\title{
Power spectrum estimation of complex signals and its application to Wigner-Ville distribution: A group delay approach
}

\author{
S V NARASIMHAN * , E I PLOTKIN and M N S SWAMY \\ Centre for Communication and Signal Processing, Department of Electrical and \\ Computer Engineering, Concordia University, 1455, De Maisonneuve Blvd. \\ West, Montreal, H3G 1M8, Canada \\ ${ }^{*}$ Present address: Aerospace Electronics Division, National Aerospace Labs, \\ Bangalore, 560017 , India \\ e-mail: svnarsim@nalsic.ernet.in
}

\begin{abstract}
In this paper, a method of estimating the power spectrum of a complex signal based on the Group Delay function (GD) is proposed and also applied to the Wigner-Ville Distribution (WVD) to reduce the ripple effect due to the truncation of the autocorrelation sequence. The proposed method is realised by the GD for a complex signal and the modified GD concept. This extends the performance advantages of the modified GD applicable to a real signal, to a complex one. Further, its application to WVD, reduces the truncation/ripple effect without sacrificing any frequency resolution, as no common window function is used. Significant improvement in performance, in terms of reduction in variance without any compromise on resolution and higher noise immunity, has been found over those of the periodogram and windowed WVD.
\end{abstract}

Keywords. Complex signals; power spectrum estimation; group delay approach; Wigner-Ville distribution; Gibb's ripple effect.

\section{Introduction}

Generally, spectral estimation aims at extracting information about the system, from its observed output (in the absence of the input), and a signal, when it is associated with noise. A good spectral estimator, for a given length of data, provides an estimate which has as high a resolution and as low a bias and variance as possible. These factors depend on the signal scenario (data length, window and the associated noise). The variance of the basic periodogram is large, though it has good resolution, low bias and good signal detectability even at high noise levels. The windowed PSE reduces the spectral variance. This variance reduction is due to smearing of the signal spectrum with the broad main 
lobe of the spectrum of the window and this results in a poor resolution. Also, only at the cost of resolution, the averaged PSE provides a lower variance, depending upon the number of segments averaged and the window used, for a given length of data. The modelbased parametric methods (Kay 1988), even for a relatively short data length, provide high resolution and low variance. But this is valid only when the signal is matched to the assumed model and the signal to noise ratio is high.

Though the power spectral density (PSD) based on the Fourier transform (FT) gives spectral information, it does not explicitly provide a time reckoning, and according to this, the spectral content is the same for ever. To analyse nonstationary signals, shorttime FT, which gives spectral information about the data within a window at its location, was introduced and this involves a tradeoff between the time localization and frequency resolution. To alleviate this, the Wigner-Ville distribution (WVD), the time-frequency representation (TFR) was introduced (Cohen 1989). The WVD, at any time instant, is the FT of the instantaneous autocorrelation (IACR) sequence of infinite lag length. Therefore, theoretically, it has infinite resolution both in time and frequency. However, practically, it is the Pseudo WVD (PWVD) that is computed which considers IACR only for a finite number of lags. In PWVD, in order to overcome the abrupt truncation effect, viz. the ripples (Gibb's effect) in the TFR along the frequency axis, the IACR is weighted by a common window function and for a given lag length, this deteriorates the frequency resolution.

The group delay function (GD), the negative derivative of phase, is attractive as it provides improved resolution over that of the PSE and facilitates ease of processing (Yegnanarayana et al 1984). However, spectral estimation based on smoothed GD, like PSE, also suffers from smearing and the reduction in variance is only at the expense of resolution. Recently, a modification for the GD, which not only preserves the advantages of the PSE, in particular, the good frequency resolution, but also reduces the variance of the estimate, has been proposed (Murthy \& Yegnanarayana 1991; Yegnanarayana \& Murthy 1992). This basically removes the zeros close to the unit circle, without disturbing the poles and zeros of the system or the signal (assuming the zeros if any are only damped ones). White noise or spectral ripples introduce zeros close to the unit circle. When the signal is the output of a system driven by a white noise or a signal with its associated white noise or signal spectrum associated with ripples due to signal truncation, the variance is due to the zeros close to the unit circle, irrespective of their origin. Consequently, reduction in the effect of only these zeros results in reduced variance without compromising on frequency resolution.

In this paper, modification for the GD has been applied to a complex signal and is achieved by considering the GD for a complex signal (Reddy \& Rao 1987). Also, for the WVD, the IACR being an analytic complex signal, the modified group delay function for a complex signal (GDCM) approach has been tailored to remove the ripple effect without applying any common window, to achieve preservation of the frequency resolution and better noise immunity. 


\section{Modified magnitude group delay function of a complex signal (MGDCM)}

\subsection{Group delay function for a complex signal}

If $x(n)$ is a minimum phase signal, then (Reddy \& Rao 1987)

$$
\begin{aligned}
\ln |X(\omega)| & =\sum_{n=0}^{\infty}\left[c_{R}(n) \cos \omega n+c_{I}(n) \sin \omega n\right], \\
\theta(\omega) & =\sum_{n=0}^{\infty}\left[-c_{R}(n) \sin \omega n+c_{I}(n) \cos \omega n\right],
\end{aligned}
$$

where $\theta(\omega)=\theta_{v}(\omega)+2 \pi I(\omega), \theta_{v}(\omega)$ is the principal value of the phase, $\theta(\omega)$ is the unwrapped phase and $c(n)=c_{R}(n)+j c_{I}(n)$ are the complex cepstral coefficients. $R$ and $I$ refer to the real and imaginary parts. For a minimum phase signal, the logmagnitude spectrum and the phase are related by a single set of complex cepstral coefficients. The GD $\tau_{m}(\omega)$ is given by

$$
\begin{aligned}
\tau_{m}(\omega) & =-\partial \theta(\omega) / \partial \omega \\
& =\sum_{n=0}^{\infty}\left[n c_{R}(n) \cos \omega n+n c_{I}(n) \sin \omega n\right] .
\end{aligned}
$$

$c_{R}(n)$ and $c_{I}(n)$ are derived from the magnitude and hence $\tau_{m}(w)$ is called the magnitude GD for a complex signal (MGDC). Also, (3) is

$$
\tau_{m}(\omega)=(1 / 2) F T\left[\left(n c(n)-n c^{*}(-n)\right)\right],
$$

where $*$ indicates conjugate operation. If $n c(n)$ is conjugate symmetric, then

$$
\tau_{m}(\omega)=F T[n c(n)] .
$$

For a mixed phase signal, the logmagnitude and phase are not related by a single set of cepstral coefficients and two different cepstral sequences $c_{1}(n)=c_{1 R}(n)+j c_{1 I}(n)$ and $c_{2}(n)=c_{2 R}(n)+j c_{2 I}(n)$, for magnitude and phase respectively, are defined. $c_{1}(n)$ and $c_{2}(n)$ are the complex cepstral coefficients of minimum phase signals derived from magnitude and phase respectively. For this case, the magnitude group delay (MGDC) is the same as given by (3) (using $c_{1}(n)$ instead of $c(n)$ ); however, the Phase group delay (PGDC) is different and is given by

$$
\tau_{p}(\omega)=\sum_{n=0}^{\infty}\left[n c_{2 R}(n) \cos \omega n+n c_{2 I}(n) \sin \omega n\right] .
$$

To compute, $\tau_{p}(\omega)$, from $(6), c_{2}(n)$ has to be derived from the phase function $\theta(\omega)$ which is an unwrapped one. But $\tau_{p}(\omega)$ can be directly computed from the FTs $X(\omega)$ and $Y(\omega)$ of the signals $x(n)$ and $y(n)=n x(n)$, respectively, by

$$
\tau_{p}(\omega)=-\left[X_{R}(\omega) Y_{R}(\omega)+X_{I}(\omega) Y_{I}(\omega)\right] /|X(\omega)|^{2}
$$

It is important to note that the computation of GD from the phase information, using (7) is valid both for real and complex signals. However, for a complex signal, the computation 
of GD from the magnitude spectrum is different from that for a real signal. Further, for a minimum phase signal, as the MGDC and PGDC are same, the above expression for PGDC can be used for computing the MGDC.

\subsection{The modified group delay for a complex signal}

The variance of a spectral estimate is due to the undesired fine structure. It is of interest to capture the spectral envelope and discard the fine structure without affecting the former. The zeros close to the unit circle manifest as spikes in the GD. Further, the polarity of the spikes depend upon whether the zero is inside or outside the unit circle. It is these spikes which contribute significantly to the fine structure of the spectrum and their effect cannot be removed by normal smoothing without the loss of resolution. The modification suggested by Murthy \& Yegnanarayana (Murthy \& Yegnanarayana 1991; Yegnanarayana \& Murthy 1992) removes these spikes effectively. Presently, it is proposed to apply this modification to the MGD for complex signals (MGDC).

Assuming that the signal under consideration $x(n)$ is generated by a complex all-pole system, driven by a complex white noise or has complex sinusoids with complex white noise and, further, that its spectrum $X(w)$ can be put in a rational form $N(\omega) / D(\omega)$, the denominator corresponds to the system or sinusoids and the numerator corresponds to the excitation or the associated noise, respectively. For this case, the MGDC is given by

$$
\tau_{m}(\omega)=\tau_{m N}(\omega)-\tau_{m D}(\omega)
$$

where $\tau_{m N}(\omega)$, and $\tau_{m D},(\omega)$, are the MGDCs for $N(\omega)$ and $D(\omega)$ respectively. Also, $\tau_{m}(\omega)$ is given by $(7)$, where $x(n)=x_{m}(n)$ and $y(n)=y_{m}(n)=n x_{m}(n), x_{m}(n)$ being the minimum phase equivalent of $x(n)$. It can be shown that

$$
\tau_{m}(\omega)=\frac{\alpha_{N}(\omega)}{|N(\omega)|^{2}}-\frac{\alpha_{D}(\omega)}{|D(\omega)|^{2}}
$$

$\alpha_{N}(\omega)$ and $\alpha_{D}(\omega)$ are the numerators of $(7)$ for $\tau_{m N}(\omega)$ and $\tau_{m D}(\omega)$ respectively. $\tau_{m N}(\omega)$ will have large amplitude spikes due to the very small values of $|N(\omega)|^{2}$, near the zeros which are close to the unit circle, while this is not so with $\tau_{m} D(\omega)$, as the roots of $D(\omega)$ are well within the unit circle. Hence, in $\tau_{m}(\omega)$, the effect of excitation or the associated noise (both have zeros near the unit circle) masks the system or the signal component which is assumed to be an all-pole one. The effect of these zeros could be reduced by multiplying $\tau_{m}(\omega),(9)$, by $|N(\omega)|^{2}$. Further, as the envelope of $|N(\omega)|^{2}$ is nearly flat, the significant features of $\tau_{m D}(\omega)$ continue to exist, with $|N(\omega)|^{2}$ fluctuations superimposed on it. Hence, the modified MGDC (MGDCM) $\tau_{m o}(\omega)$, is

$$
\tau_{m o}(\omega)=\tau_{m}(\omega)|N(\omega)|^{2}
$$

For the computation of MGDCM, an estimate of $N|(\omega)|^{2},|\hat{N}(\omega)|^{2}$ is required in addition to the MGDC. This is achieved by computing the ratio of the signal power spectrum, $|X(\omega)|^{2}$, to the smoothed power spectrum of the signal, $|\bar{X}(\omega)|^{2}$, obtained by the truncated cepstral coefficient sequence. The values of $|N(\omega)|^{2}$ around the zeros have to be preserved so that it cancels small values in the denominator of the first term of (9). Therefore, the estimate $|\hat{N}(\omega)|^{2}$ should retain all the sharp fluctuations of the logmagnitude spectrum and should have a flat spectral envelope. 


\section{The Wigner-Ville distribution and the modified magnitude group delay for a complex signal}

For a signal $x(t)$, WVD is defined as

$$
W_{x}(t, \omega)=\int_{-\infty}^{\infty} x(t+\tau / 2) x^{*}(t-\tau / 2) e^{-j \omega \tau} \mathrm{d} \tau,
$$

where $r(\tau)=\left[x(t+\tau / 2) x^{*}(t-\tau / 2)\right]$ is the instantanec'is autocorrelation function and $*$ indicates conjugate operation. For computational purposes, it is necessary to weigh the signal by a window before evaluating the WVD and this window slides along the time axis. For a window function, $h(t), h(t)=0$ for $|t|>T / 2$, the WVD of the windowed signal is

$$
W_{x h}(t, \omega)=\int_{-\infty}^{\infty} W_{x}(t, \xi) W_{h}(t-\xi) \mathrm{d} \xi,
$$

where $W_{h}(t, \omega)$ is the WVD of the window function. This WVD of the windowed signal is called Pseudo Wigner-Ville Distribution $(P W V D), P W_{x}(t, \omega)$. The effect of the window is to smear the WVD along the frequency axis. For a real symmetrical window, the PWVD is the FT of the windowed function $\left[x(t+\tau / 2) x^{*}(t-\tau / 2)\right]$, the window being $h^{2}(\tau / 2)$. The window eats away the correlation function at higher lags which results in poor spectral resolution.

The quadratic operation on the signal, causes the WVD to be bilinear and this introduces crossterms for multicomponent signals (Flandrin 1984; Velez \& Absher 1990). The crossterm appears midway between every two components of the signal. Its amplitude is proportional to the product of the two components' amplitudes and it oscillates in time at a frequency equal to the frequency separation between them. The crossterm effect, which makes the interpretation of the WVD difficult, can be reduced by smoothing the WVD along the time axis. The smoothing process, in time for crossterms and in frequency for the lag window, can be considered a two-dimensional convolution of the WVD of the signal with that of the smoothing kernel, $\Phi(t, \omega)$ (Flandrin 1984). Using different smoothing kernels, a class of distributions known as Cohen's class (Cohen 1989) can be realized. The kernel determines the properties of the distribution (Jeong \& Williams 1992). The properties, viz. marginality in frequency, instantaneous frequency and frequency support, are not satisfied for common windows due to smearing.

The very definition of the WVD demands the signal to be sampled at twice the Nyquist sampling rate, otherwise it introduces aliasing in the frequency domain as the periodicity is $\pi$ instead of $2 \pi$. This is overcome by using an analytic signal (Picone 1988) which necessitates further processing techniques to handle complex signals.

In the computation of PWVD, for a given lag length, the lag windowing used to take care of the truncation effects eats away the higher IACR lags and reduces the frequency resolution relative to that of no windowing. Further, the very definition of the WVD, which results in an aliasing in the frequency domain, demands the conversion of a real signal to a complex analytic signal. With the MGDCM, as the zeros close to the unit circle are removed without applying any window, the loss in the frequency resolution which would have occurred due to windowing can be preserved. Hence, the MGDCM developed in $\S 2$, can be used for the WVD to preserve its original resolution. Also, as the MGDCM removes 
the zeros due to the associated white noise, the proposed WVD indirectly achieves better noise immunity.

For the MGDC computation, the starting point is the signal under consideration, as mentioned in $\$ 2.1$. However, to apply the MGDC to the present TFR, the IACR smoothed along the time axis (SIACR) is the beginning point and the magnitude estimate required, (1), at a particular time instant is obtained from the FT of the SIACR at that instant, the WVD slice estimate. This estimate, at a frequency, is supposed to be a positive quantity as it represents the power spectral density (PSD). However, due to the inevitable presence of the rectangular window, the values may become negative. This is due to the ideal PSD getting convolved with the FT of the rectangular window. Presently, as the computation of the MGDC $\tau_{m}(\omega)$, involves logarithmic operation, ((1) and (3)), it is necessary to ensure that the FT of the SIACR is positive. This is achieved by raising the floor level by sufficiently scaling up the SIACR at zeroth lag. Further, in computing $\tau_{m}(\omega)$ the equivalent magnitude spectrum is computed from the PSD and the linearly weighted cepstral coefficient sequence is made conjugate symmetric.

According to (10), the MGDCM, $\tau_{m o}(\omega)$, is computed by using an estimate of $|N(\omega)|^{2}$ and this estimate is $[X(\omega) / \bar{X}(\omega)]^{2}, \bar{X}(\omega)$ being the cepstrally smoothed spectrum obtained by the truncated cepstral sequence of $x(n)$. That is,

$$
\hat{N}(\omega)=X(\omega) / \bar{X}(\omega)=1+[\Delta(\omega) / \bar{X}(\omega)]
$$

Here, $\Delta(\omega)$ represents the fluctuating part of $X(\omega)$. By multiplying $\tau_{m}(\omega)$, by $|\hat{N}(\omega)|^{2}$, a spectrum which is free from contribution due to input or the associated noise and having improved resolution is obtained and this is different from $\bar{X}(\omega)$. For a signal having a flat spectral characteristic, in the GD $\tau_{m}(\omega)$, the contribution is only due to $\Delta(\omega)$. Therefore, to get a $\tau_{m o}(\omega)$, which is free from fluctuations, $\tau_{m}(\omega)$ has to be multiplied by $|\Delta(\omega)|^{2}$,

$$
\tau_{m o}(\omega)=\tau_{m}(\omega)|\Delta(\omega)|^{2}
$$

Presently, it is required to remove the ripple on the floor, which is equivalent to a flat spectral characteristic and the pedestal height is immaterial as it is not reflected in the GD. The PSD which is free from the ringing/ripple effect and has better resolution is obtained from $\tau_{m o}(\omega)$, using (14) and by retracing the MGDC computation procedure in the reverse order ((3) and (1)). It is important to note that in obtaining the processed PSD, the cepstral coefficient sequence has to be made conjugate symmetric. If only the resolution is of importance, then only the positive values of the $\tau_{m o}(\omega)$ above a certain threshold can be considered. To achieve the improved WVD, these operations have to be repeated for every sample. It is important to note that since no window is applied, the proposed method obeys frequency domain properties better than the PWVD.

\section{Simulation results}

\subsection{Performance of the $M G D C M$}

The performance of the power spectrum estimation using the MGDCM for complex signals is illustrated both for a complex autoregressive process generated by driving a complex 
AR system by a white Gaussian noise of unit variance which is also complex, and for complex sinusoids in complex white Gaussian noise. The AR process and the complex sinusoids are the same as those considered by (Yegnanarayana \& Murthy 1992), except that the former is a complex version.

The AR system considered has roots at $(0.6321+j 0.7593)$ and $(0.7569+j 0.6204)$. Further, the same AR process which is associated with complex white Gaussian noise having a signal-to-noise ratio (SNR) equal to $0 \mathrm{~dB}$ is considered. For sinusoids plus white noise,

$$
x(n)=\sqrt{10} e^{j 2 \pi(0.10) n}+\sqrt{20} e^{j 2 \pi(0.15) n}+u(n),
$$

is considered the signal. $u(n)$ is the additive complex white Gaussian noise, and its variance is adjusted to get SNRs equal to $20 \mathrm{~dB}$ and $0 \mathrm{~dB}$. For the AR process and for the sinusoids plus noise, the data lengths used are 256 and 100 points respectively. For both the cases, in computing the $|N(\omega)|^{2}$ estimate, the smoothed spectrum is obtained by considering the initial 3 cepstral coefficients.

The performance of the MGDCM for the AR signal and for the sinusoids considered, at $\mathrm{SNR}=0 \mathrm{~dB}$, are shown in figures 1 and 2 respectively. Further, these results are compared with those of the PSE. In figures 1 and 2, (a) and (c) correspond to individual estimates; (b) and (d) show the mean and variance of the estimate obtained by 50 realisations. The individual estimate obtained by MGDCM is free from the fine structure due to the input and the associated noise. The variance plots shown also support this as the PSE variance is very high compared to those of MGDCM. Further, the reduction in variance achieved by the proposed method over the PSE is quantified by computing the Sum Sample Variance Ratio (SSVR) given by SSVR $=\sum V_{\tau}(\omega) / \sum V_{P}(w)$, where $V_{\tau}(\omega)$ stands for the variance computed by the GD approach and $V_{P}(\omega)$ for that by PSE. For the AR process and for sinusoids, the SSVR has values of 0.1783 and 0.0438 respectively. The mean plots are similar, except that the mean obtained by the PSE has still some fine fluctuations, even after averaging 50 estimates. For the clean AR process and for the sinusoids with noise $(S N R=20 \mathrm{~dB}$ ), the SSVR values are 0.7471 and 0.0126 respectively. The reduction in variance achieved is more significant for sinusoids than for the AR process.

The smoothing parameter, the number of cepstral coefficients used, determines the variance of the proposed estimators and to get a good variance reduction, this number should be kept as small as possible. Compared to the PSE, the proposed estimator has a significantly low variance. Further, depending upon the signal and its associated noise, its variance ranges only from $70 \%$ to $2 \%$ of that of the PSE and the reduction seems to be more effective when the SNR is low.

\subsection{Performance of the improved WVD}

The performance of the proposed method is illustrated for both FSK and chirp signals. As required by the WVD, the signals are converted to analytic signals by computing the Hilbert transform of the original real signal. The Hilbert transform has been realised by convolving the signal with the impulse response of the Hilbert transformer (Picone, 1988) (weighted by a Kaiser window with a smoothing factor of 8), in time domain. 
(a)

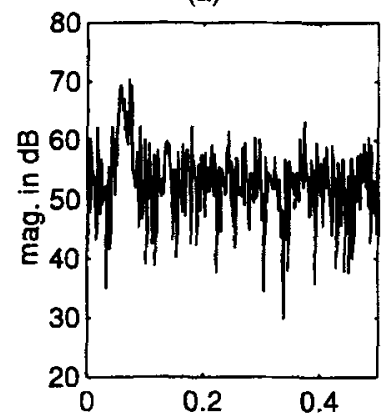

(b)

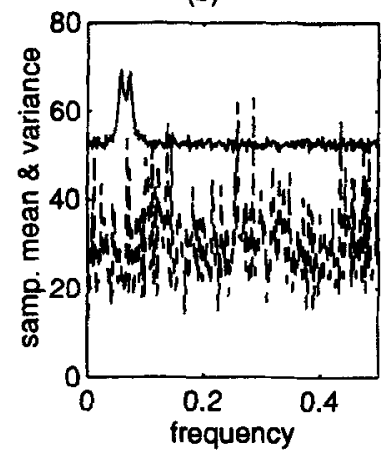

(a)

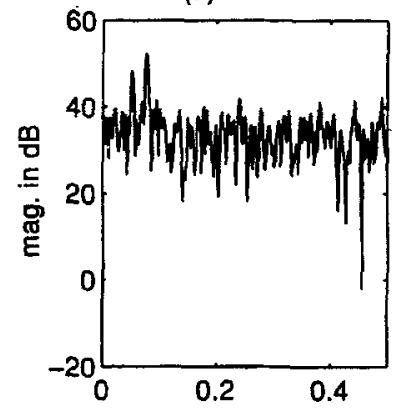

(b)

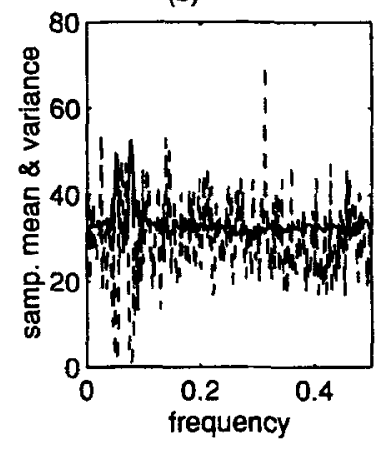

(c)

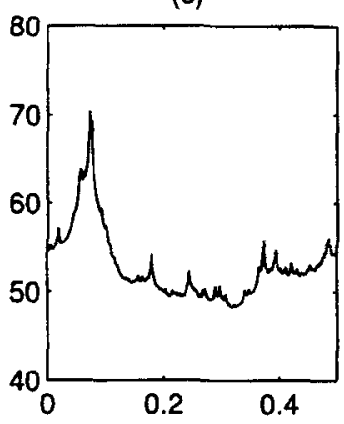

(d)

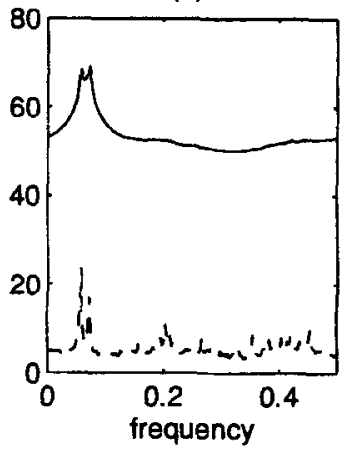

(c)

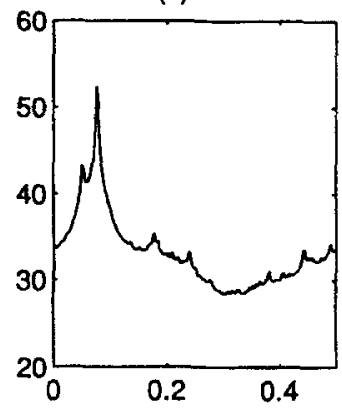

(d)

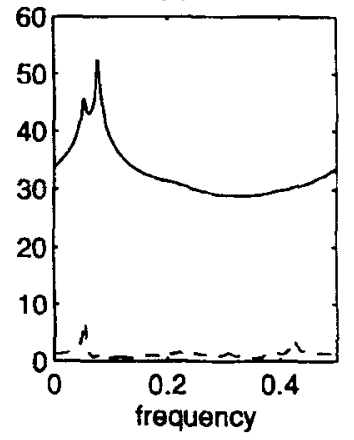

Figure 1. Performance of MGDCM for the AR process with white noise $(\mathrm{SNR}=0 \mathrm{~dB})$. Individual estimates by periodogram (a) and MGDCM (c). Mean (-) and variance (--) by periodogram (b) and MGDCM (d).

Figure 2. Performance of MGDCM for the sinusoids plus white noise (SNR = $0 \mathrm{~dB}$ ). Individual estimates by periodogram (a) and MGDCM (c); mean (-) and variance $(--)$ by periodogram (b) and MGDCM (d). 

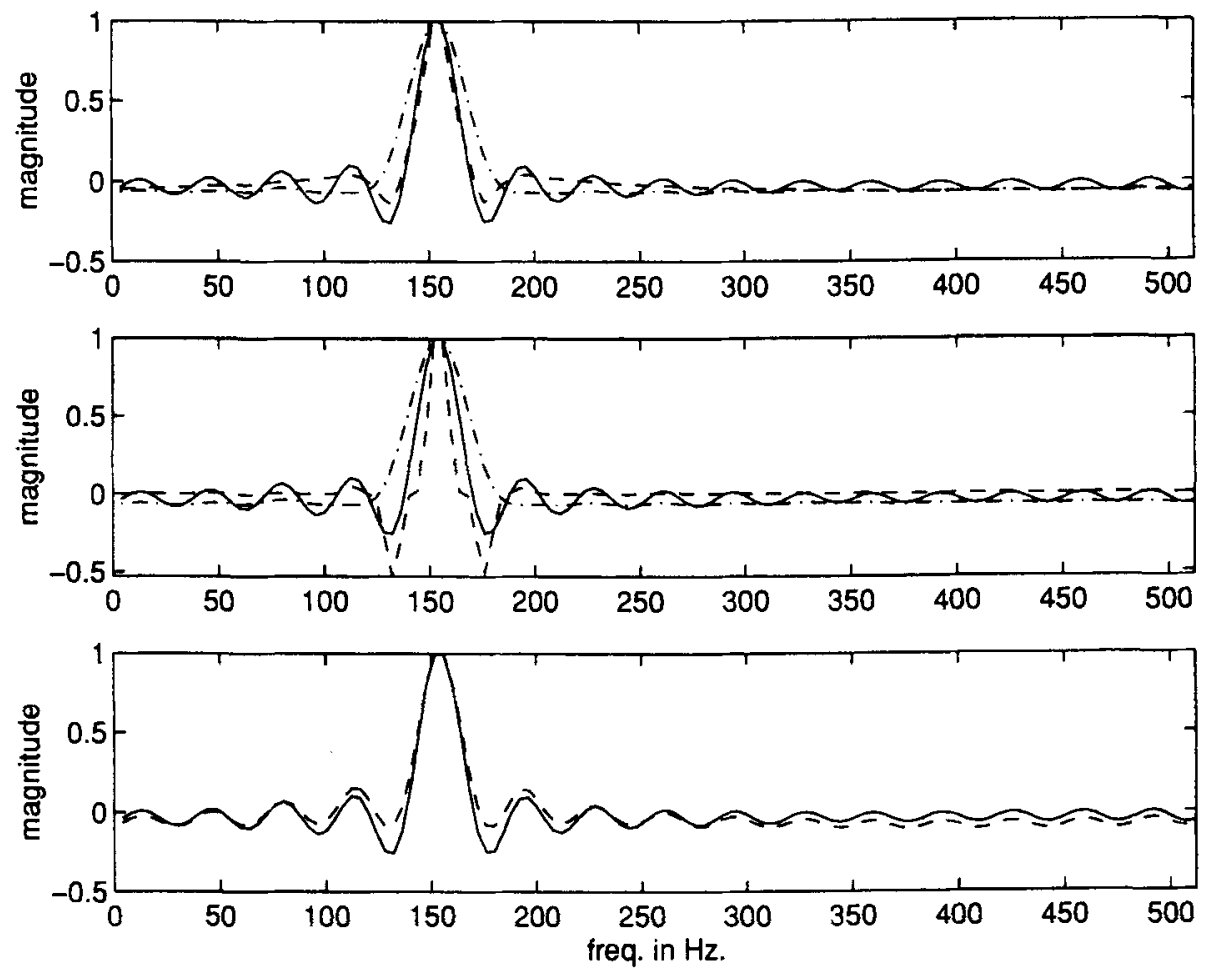

Figure 3. Comparison of the resolution and ripple reduction by PWVD and MGDCM. (a) WVD slice by: - rectangular window (RW), --- Hamming, - - MGDCM, (b) WVD slice by: - RW, --- Hamming, -- MGDCM, (c) WVD slice by: - RW and -- MGDCM, using $|\hat{N}(\omega)|^{2}$.

For the FSK signal, the number of lags considered is 31 . To reduce the crossterms, a 5-point boxcar smoothing is applied along the time axis, for the autocorrelation function at each lag. Further, a discrete FT of length 128 points is used. For the PWVD, the SIACR is weighted by a Hamming window. For the proposed method, to avoid the negative spectral values in the PSD, SIACR at zeroth lag has been lifted by a factor of 100 . The $|\Delta(\omega)|^{2}$ estimate is obtained by considering the initial 8 cepstral coefficients in computing the smoothed PSD.

To get a comparative performance view, a section of the TFR obtained by the proposed method and by the PWVD, corresponding to the same instant of time, are shown in figure 3. This brings out the ripple reduction ability and the frequency resolution preserving ability of the proposed method (figure 3a). Further, in the MGDCM domain, a better improvement in frequency resolution is observed (figure $3 b$ ) and this is due to the very nature of the GD. The use of the $|N(\omega)|^{2}$ estimate is unable to remove the ripple (figure $3 c$ ) and this is due to the fact that the fluctuations in it are different from what is required to be canceled in the MGDC (for the ripple on the floor).

The TFR obtained by the proposed method removes ripples/ringing due to abrupt truncation and at the same time preserves the resolution of the rectangular window (figure $4 \mathrm{~b}$ ). Hence, it has a superior frequency resolution than the PWVD which uses a Hamming window (figure 4a). However, the residual crossterms left after smoothing also get enhanced. 


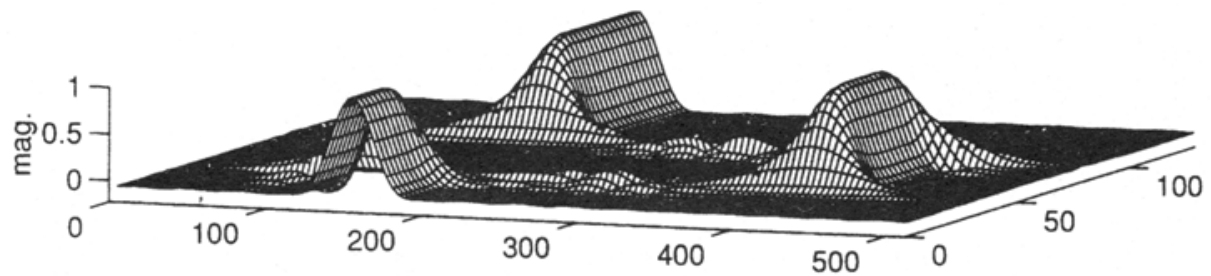

(a)

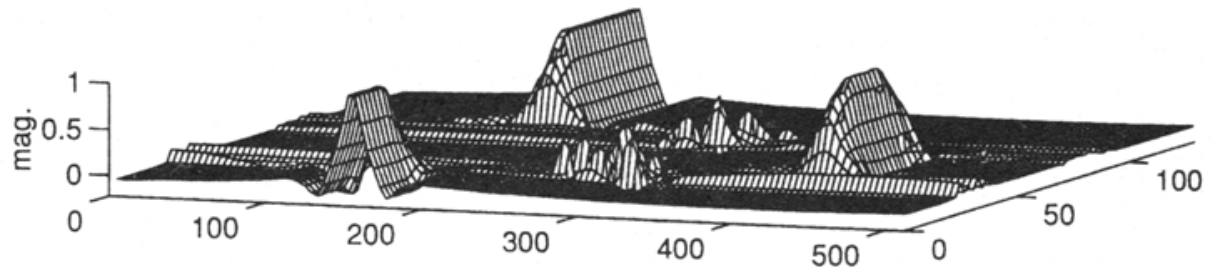

(b)

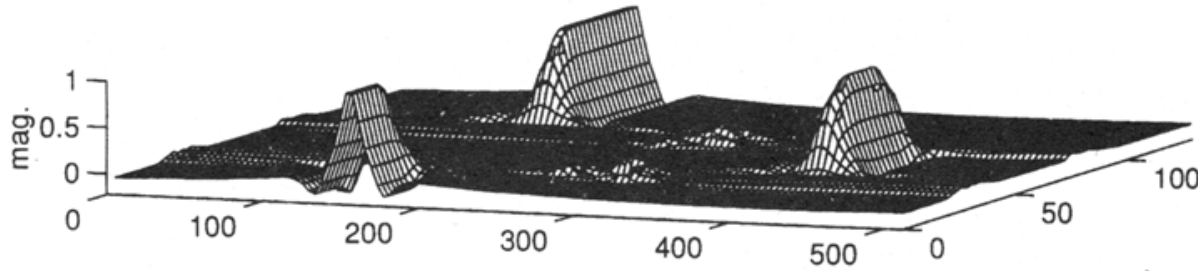

(c)

freq. in $\mathrm{Hz}$.
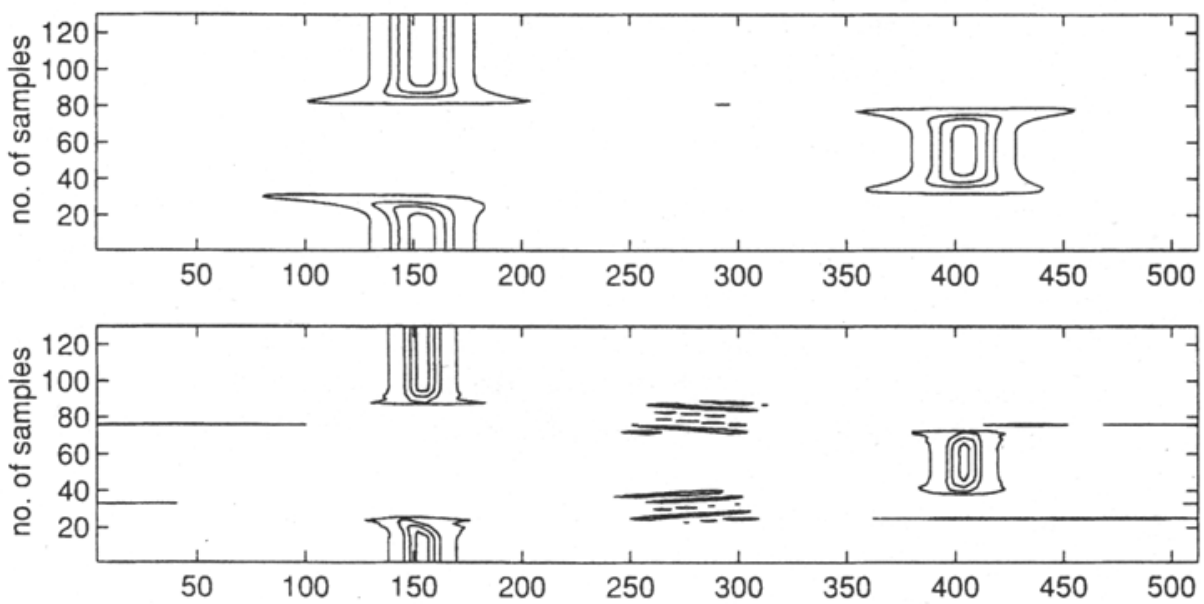

(e)

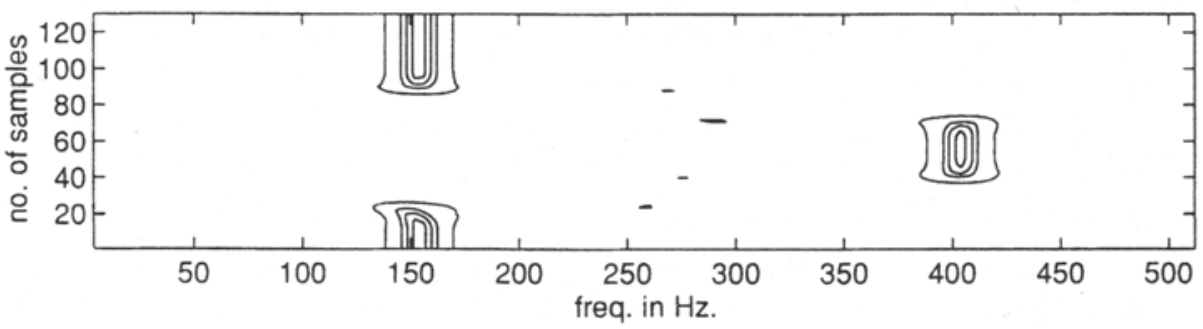

Figure 4. TFR representation for FSK signal by (a) PWVD, (b) MGDCM and (c) MGDCM after second smoothing. (d). (e) and (f) Contour plots for (a), (b) and (c) respectively. 


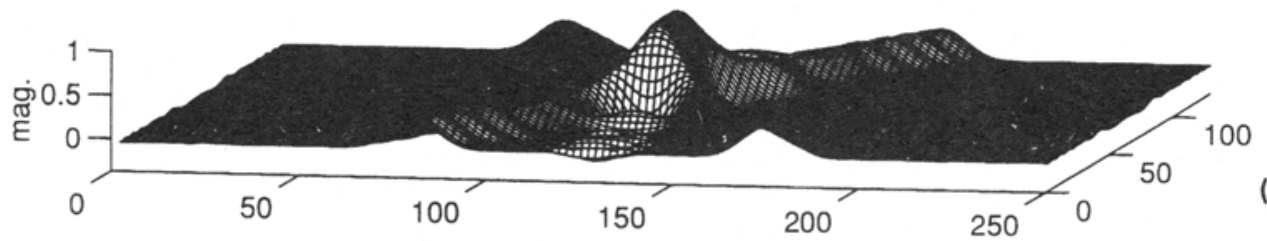

(a)
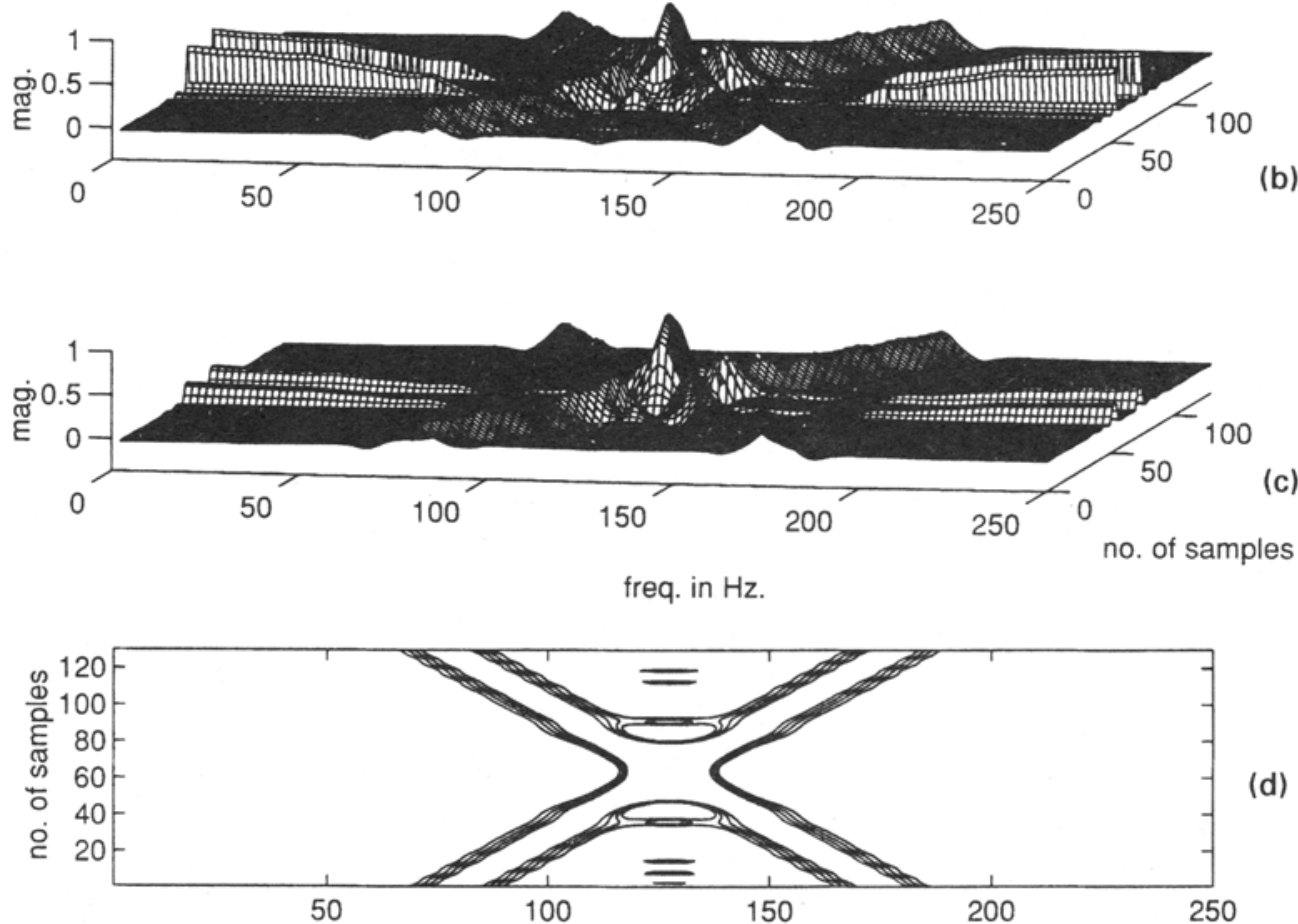

(d)

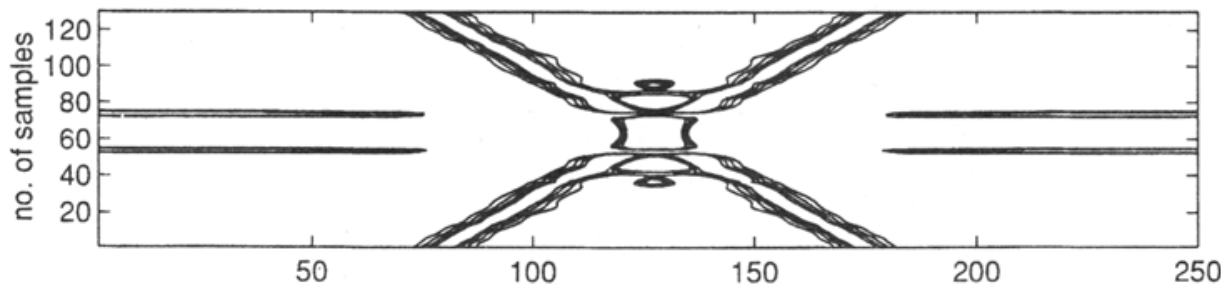

(e)

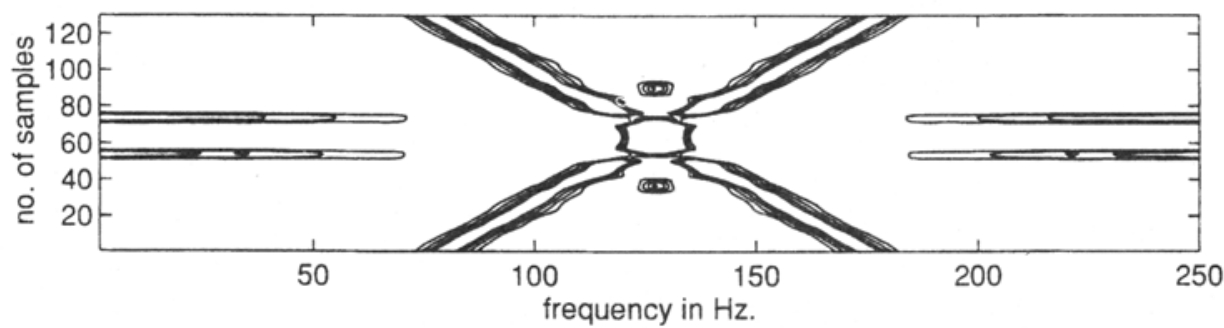

Figure 5. TFR representation for chirp signal by (a) PWVD. (b) MGDCM and (c) MGDCM after second smoothing. (d). (e) and (f) Contour plots for (a). (b) and (c) respectively. 

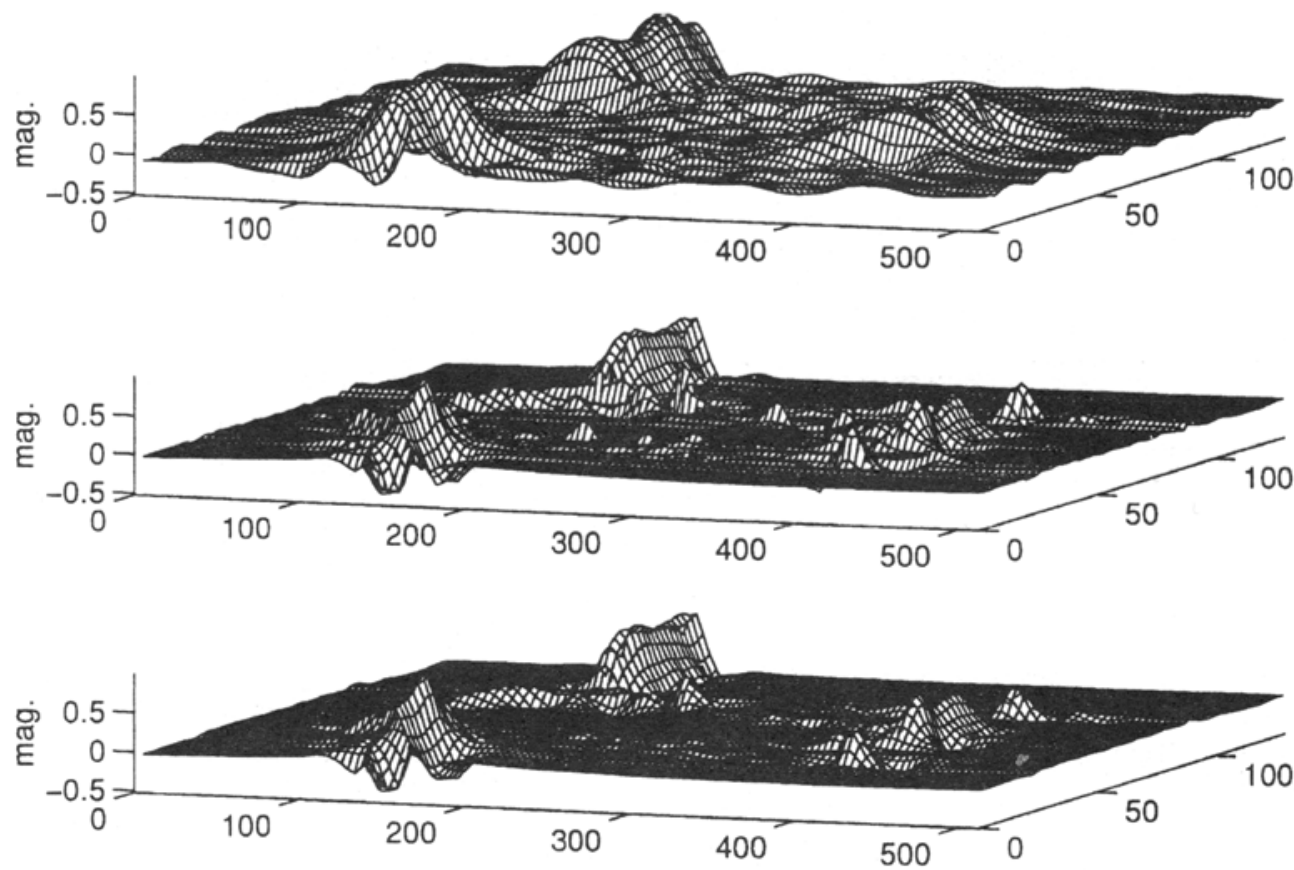

(c)

no. of samples

freq. in $\mathrm{Hz}$.

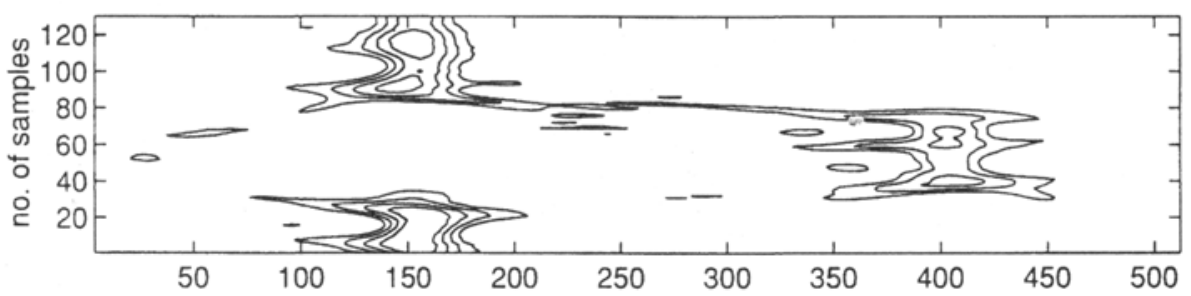

(d)

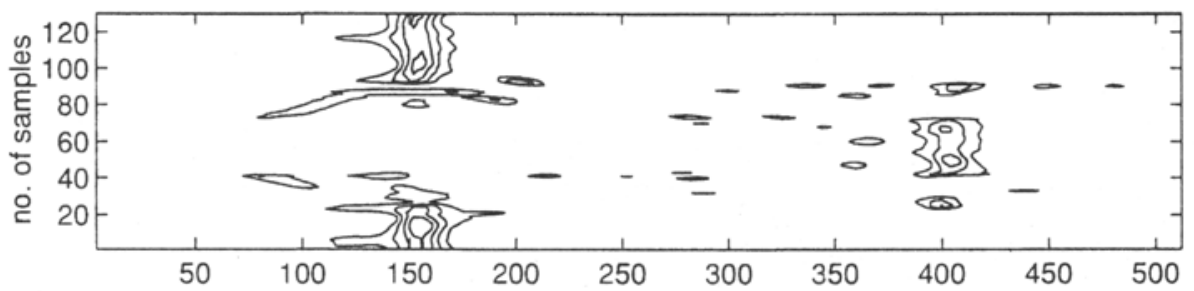

(e)

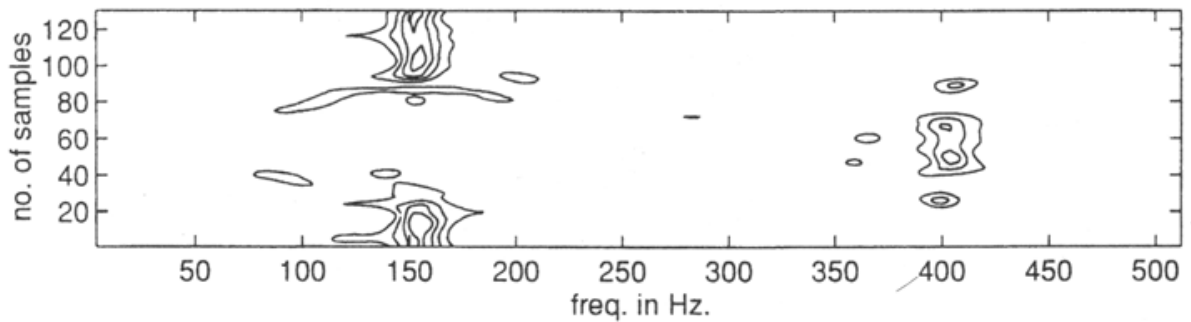

(f)

Figure 6. TFR representation for FSK signal with white noise by (a) PWVD, (b) MGDCM, and (c) MGDCM after second smoothing. (d), (e) and (f) Contour plots for (a), (b) and (c) respectively. 


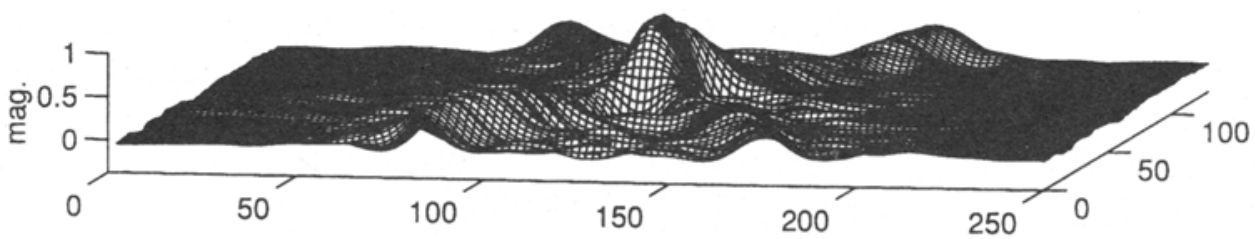

(a)

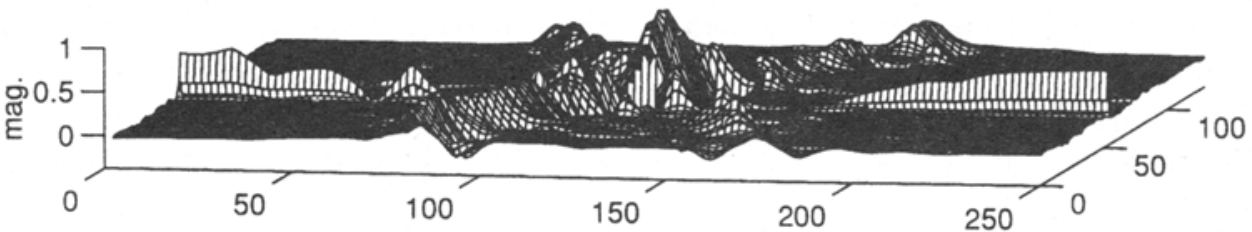

(b)

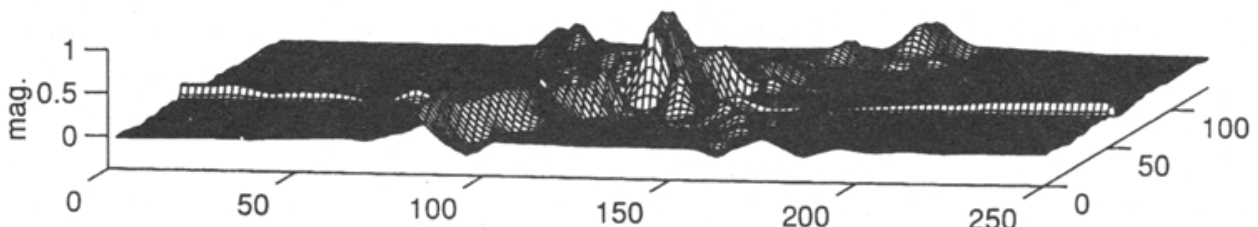

(c)

freq. in $\mathrm{Hz}$.

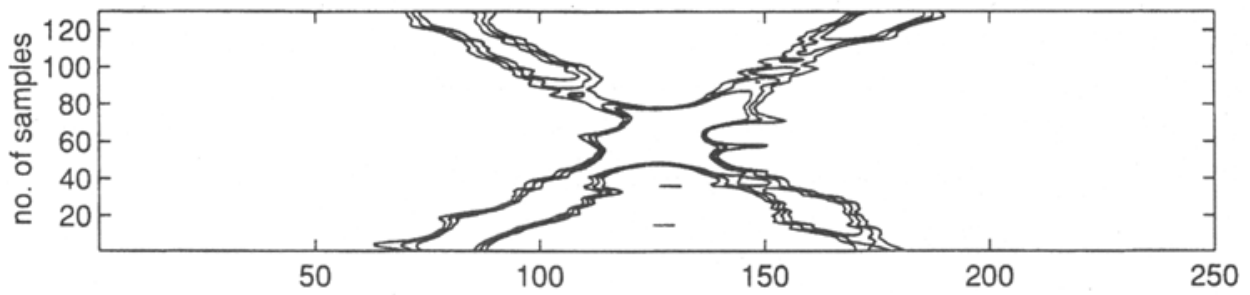

(d)

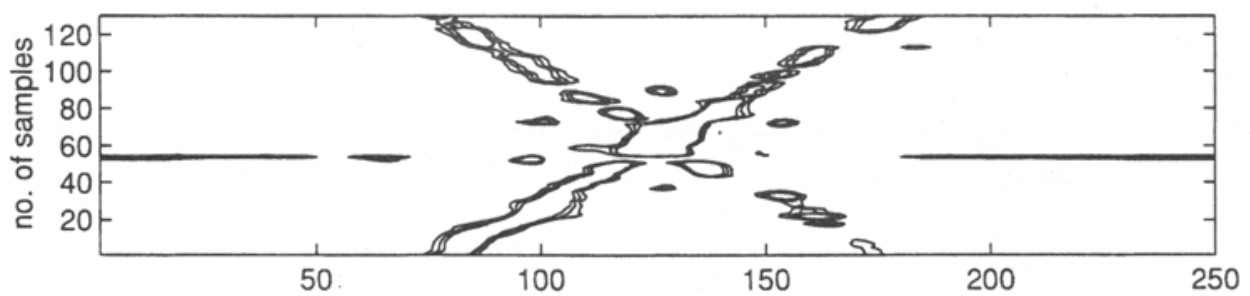

(e)

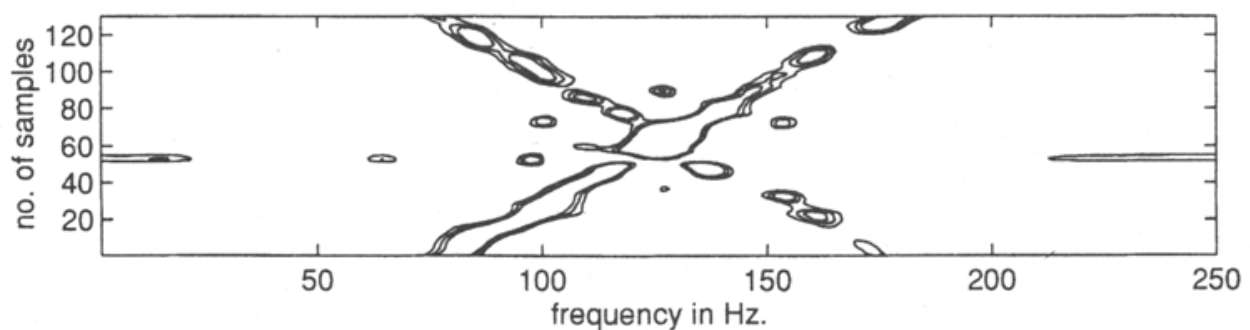

(f)

Figure 7. TFR representation for chimp signil with white noise by a) PWVD, (b) MGDCM and (c) by MGDCM after second smoothing. (d), (e) and (f) Contour plots for (a), (b) and (c) respectively. 
This crossterm effect can be significantly reduced by a second smoothing in time (figure $4 \mathrm{c}$ ). The contour plots corresponding to figure $4 \mathrm{a}-\mathrm{c}$ are shown in figure $4 \mathrm{~d}-\mathrm{f}$, respectively and they support the fact that the resolution is improved over the PWVD. If only the spectral peak location is of interest, then the GDMCM, above a certain threshold, can be plotted in the TF plane rather than the PSD. This provides additional frequency resolution (figure 3b).

For the linear chirp signal, the results obtained are shown in figure 5. The IACR is smoothed along the time axis by a 9-point boxcar window. For this case also, the zeroth lag is increased by a factor 100 . A floor level above a minimum value does not affect the performance. However, increasing the floor level by a very large amount may affect the TFR estimation. For the estimation of $|\Delta(\omega)|^{2}$, the first 8 cepstral coefficients are used in computing the smoothed PSD. Figure $5 \mathrm{c}$ shows the TFR obtained by the proposed method with a second time smoothing (box car of 5 points). The crossterm effect was less when compared to that of PWVD even prior to the second smoothing (figure 5b). However, the second smoothing helped in reducing the ridge type of effect which occurs at the region of crossing of the two chirps (figure $5 \mathrm{c}$ ). The reduction in the crossterms may be due to their nature and as they are similar to ripples along the frequency axis, they are flattened. In this case also, the frequency resolution is preserved and the ripple effect is reduced. The contour plots shown in figure $5 \mathrm{~d}-\mathrm{f}$; support these results.

The performance of the proposed method, for the above signals in the presence of noise at $\mathrm{SNR}=5 \mathrm{~dB}$, is shown in figures 6 and 7 respectively. In both cases, for the proposed method, the spectral peaks due to noise are reduced relatively to those of the PWVD. This is expected as the MGDCM not only removes the zeros due to the ripple effect but also the zeros due to noise and it does not distinguish between the two.

\section{Conclusions}

A method was proposed for estimating the power spectrum for a complex signal, which combines the concepts of the GD for a complex signal and the modified GD. Further, this approach was applied to the WVD to remove the ripple effect, due to the abrupt truncation of the autocorrelation sequence, by tailoring the modified GD for a complex signal, MGDCM.

The proposed MGDCM method provides a spectral estimate which has a significantly lower variance than that of the periodogram, without compromising on spectral resolution. Depending upon the signal scenario, the proposed estimator has a variance ranging from about $70 \%$ to $2 \%$ of that of the periodogram. Further, as the variance reduction is more effective when the SNR is low, its immunity to noise is very significant.

The improved WVD based on the MGDCM was found to be very effective in removing the ripple effect due to truncation and in preserving the resolution of a rectangular window as no common window function is used. Further, since in addition to removing the zeros due to the spectral ripple the zeros due to white noise are also removed, it has an enhanced noise immunity. 


\section{References}

Cohen L 1989 Time-frequency distribution - A review. Proc. IEEE. 77: 941-981

Flandrin P 1984 Some features of time-frequency representation of multicomponent signals. Int. Conf. on Acoustics, Speech and Signal Processing, pp 41B.4.1-41B.4.4

Jeong J, Williams W J 1992 Kernel design with reduced interference distributions. IEEE Trans. Signal Process. 38: 402-412

Kay S M 1988 Modern spectral estimation: Theory and application (Englewood Cliffs, NJ: Prentice Hall)

Murthy H A, Yegnanarayana B 1991 Speech processing using group delay function. Signal Process. 22: $259-267$

Picone J 1988 Spectrum estimation using an analytic signal representation. Signal Process. 15: $169-182$

Reddy G R, Rao V V 1987 Group delay functions for complex signals. Signal Process. 12: 5-15

Velez E F, Absher R G 1990 Spectral estimation based on the Wigner-Ville representation. Signal Process. 20: $325-346$

Yegnanarayana B, Murthy H A 1992 Significance of group delay functions in spectrum estimation. IEEE Trans. Signal Process. 40: 2281-2289

Yegnanarayana B, Saikia D K, Krishnan T R 1984 Significance of group delay functions in signal reconstruction from spectral magnitude or phase. IEEE Trans. Acoustics, Speech Signal Process. ASSP-32: 610-623 